Multigrid methods with

Powell-Sabin splines

\author{
Hendrik Speleers \\ Paul Dierckx \\ Stefan Vandewalle
}

Report TW 488, February 2007

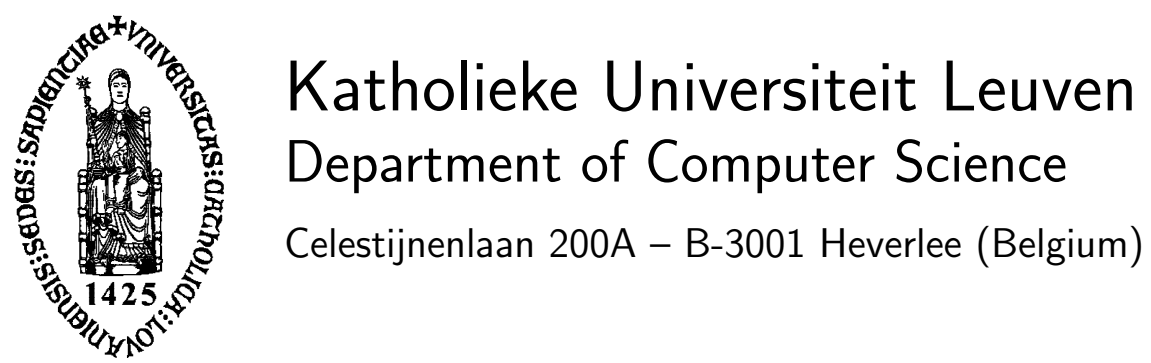




\title{
Multigrid methods with Powell-Sabin splines
}

\author{
Hendrik Speleers \\ Paul Dierckx \\ Stefan Vandewalle
}

Report TW 488, February 2007

Department of Computer Science, K.U.Leuven

\begin{abstract}
We present a multigrid algorithm for the solution of the linear systems that arise from a finite element discretization of second order elliptic partial differential equations with Powell-Sabin splines. We show that the method yields a uniform convergence independent of the mesh size.
\end{abstract}

Keywords : Powell-Sabin splines, multigrid

AMS(MOS) Classification : Primary : 65M55 Secondary : 65D07 


\title{
Multigrid methods with Powell-Sabin splines
}

\author{
Hendrik Speleers*, Paul Dierckx and Stefan Vandewalle \\ Department of Computer Science, Katholieke Universiteit Leuven \\ Celestijnenlaan 200A, B-3001 Leuven, Belgium
}

\begin{abstract}
We present a multigrid algorithm for the solution of the linear systems that arise from a finite element discretization of second order elliptic partial differential equations with Powell-Sabin splines. We show that the method yields a uniform convergence independent of the mesh size.
\end{abstract}

Keywords: Powell-Sabin splines, multigrid

AMS classification: $65 \mathrm{M} 55,65 \mathrm{D} 07$

\section{Introduction}

Spline functions are popular approximation tools in a broad range of applications. Here, we consider the use of spline functions for solving partial differential equations in a finite element approach. It is hard to determine the dimension of bivariate spline spaces on arbitrary triangulations in terms of the numbers of vertices and triangles [24]. For some particular choices of the polynomial degree and smoothness exact results are found $[1,10]$, but in general and especially for low degree polynomials the problem remains open. One can overcome this problem by using macro-elements, i.e., splitting each triangle in the mesh in a particular way. Well-known in the finite element literature is the cubic Clough-Tocher spline space [4]. Powell and Sabin [19] constructed $C^{1}$-continuous quadratic splines. They considered two families, the so-called PS 6-split and PS 12-split splines. In this paper we focus on the 6-split version, and we will refer to them as the Powell-Sabin splines.

Powell-Sabin splines can be compactly represented in a stable normalized B-spline basis [5]. This representation has an intuitive geometric interpretation involving tangent control triangles. Vanraes et al. [27] considered a global subdivision scheme, which was extended to a local subdivision scheme in [21]. These properties ensure their effectiveness in many application domains. PowellSabin splines are appropriate for surface modelling and visualization [8, 25], and data fitting $[6,28,17]$. Recently, they are also explored as finite elements for the numerical solution of partial differential equations [22]. In [15] an additive multigrid preconditioner using hierarchical PowellSabin splines is proposed for solving the discretized biharmonic equation. Oswald [18] considered a hierarchical finite element method for the biharmonic equation based on PS 12-split splines.

This paper deals with a geometric (multiplicative) multigrid approach using Powell-Sabin splines. For the general theory of multigrid methods we recommend the books $[7,2]$. Our model problem is the second order elliptic Poisson equation, i.e.,

$$
-\Delta u=f \text { in } \Omega, \quad u=0 \text { on } \partial \Omega .
$$

\footnotetext{
*Research Assistant of the Fund for Scientific Research Flanders (Belgium)
} 
The Ritz-Galerkin discretization of (1.1) with Powell-Sabin B-splines as finite elements leads to a sparse linear system [22]. As usual, simple iterative methods, e.g. the Jacobi and Gauss-Seidel method, converge very slowly for large systems since their convergence rate depends on the mesh size of the triangulation. By using a hierarchy of meshes, one can accelerate the convergence of the basic iterative method. Using the Powell-Sabin subdivision scheme, we can easily create a nested sequence of triangulations with natural intergrid transfer operators. The multigrid scheme that we propose has a uniform convergence, i.e., the number of iterations required to obtain a prescribed accuracy is bounded independent of the mesh size.

The paper is organized as follows. Section 2 recalls the definition of the Powell-Sabin spline space, and the construction of a normalized basis. It describes also the relevant aspects of Powell-Sabin subdivision. Section 3 discusses a multigrid algorithm for Powell-Sabin splines. We prove uniform convergence in the $l_{2}$-norm, and we illustrate the multigrid scheme with some numerical examples. Finally, in section 4 we end with some concluding remarks.

\section{Powell-Sabin splines}

\subsection{The space of Powell-Sabin splines}

Consider a simply connected subset $\Omega \in \mathbb{R}^{2}$ with polygonal boundary $\partial \Omega$. Assume a conforming triangulation $\Delta$ of $\Omega$ is given, consisting of $t$ triangles $\rho_{j}, j=1, \ldots, t$, and having $n$ vertices $V_{k}$, $k=1, \ldots, n$. A triangulation is conforming if no triangle contains a vertex different from its own three vertices. Denote $|\Delta|$ as the mesh size of $\Delta$, i.e., the length of the largest edge in $\Delta$.

The Powell-Sabin (PS) refinement $\Delta^{*}$ of $\Delta$ partitions each triangle $\rho_{j}$ into six smaller triangles with a common vertex $Z_{j}$. This partition is defined algorithmically as follows:

1. Choose an interior point $Z_{j}$ in each triangle $\rho_{j}$. If two triangles $\rho_{i}$ and $\rho_{j}$ have a common edge, then the line joining $Z_{i}$ and $Z_{j}$ should intersect the common edge at some point $R_{i j}$.

2. Join each point $Z_{j}$ to the vertices of $\rho_{j}$.

3. For each edge of the triangle $\rho_{j}$

(a) which is common to a triangle $\rho_{i}$ : join $Z_{j}$ to $R_{i j}$;

(b) which belongs to the boundary $\partial \Omega$ : join $Z_{j}$ to an arbitrary point $R$ on that edge.

In Figure 1(a) such a PS refinement of a given triangulation is drawn in dashed lines.

The space of piecewise quadratic polynomials on $\Delta^{*}$ with global $C^{1}$-continuity is called the PowellSabin spline space:

$$
S_{2}^{1}\left(\Delta^{*}\right)=\left\{s \in C^{1}(\Omega):\left.s\right|_{\rho_{j}^{*}} \in \Pi_{2}, \rho_{j}^{*} \in \Delta^{*}\right\} .
$$

Powell and Sabin [19] proved that the following interpolation problem

$$
s\left(V_{l}\right)=f_{l}, D_{x} s\left(V_{l}\right)=f_{x, l}, D_{y} s\left(V_{l}\right)=f_{y, l}, \quad l=1, \ldots, n .
$$

has a unique solution $s(x, y) \in S_{2}^{1}\left(\Delta^{*}\right)$ for any given set of $n\left(f_{l}, f_{x, l}, f_{y, l}\right)$-values. It follows that the dimension of the Powell-Sabin spline space $S_{2}^{1}\left(\Delta^{*}\right)$ is equal to $3 n$.

In $[13,14]$ it is shown that this spline space has optimal approximation power. Let $\|\cdot\|_{L_{p}}$ be the $L_{p}$-norm on $\Omega$, and $|\cdot|_{W_{p}^{k}}$ be the usual semi-norm in the Sobolev space $W_{p}^{k}(\Omega)$, i.e.,

$$
|v|_{W_{p}^{k}}=\sum_{\alpha+\beta=k}\left\|D_{x}^{\alpha} D_{y}^{\beta} v\right\|_{L_{p}} .
$$




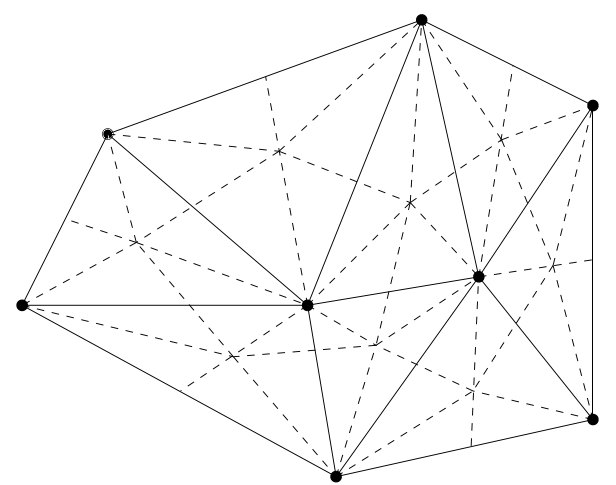

(a)

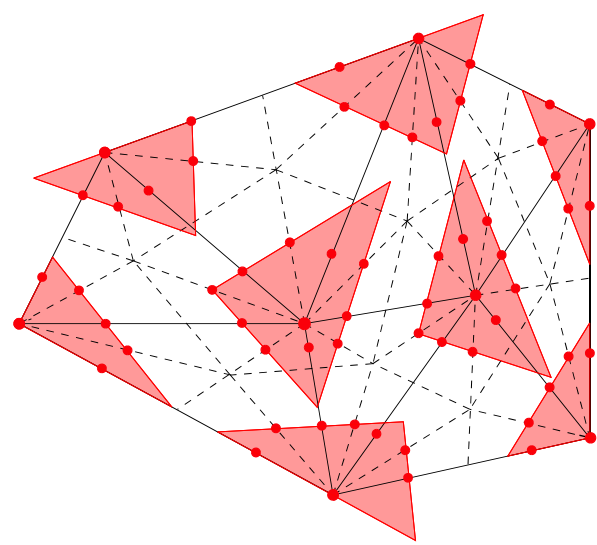

(b)

Figure 1: (a) A PS refinement $\Delta^{*}$ (dashed lines) of a given triangulation $\Delta$ (solid lines); (b) the PS points (bullets) and a set of suitable PS triangles (shaded).

Theorem 2.1 (Approximation). For every $0 \leq k \leq 2$, there exists a quasi-interpolation operator $\mathcal{Q}_{k}: W_{p}^{k+1} \rightarrow S_{2}^{1}\left(\Delta^{*}\right)$ such that

$$
\left\|D_{x}^{\alpha} D_{y}^{\beta}\left(f-\mathcal{Q}_{k} f\right)\right\|_{L_{p}} \leq K_{1}|\Delta|^{k+1-\alpha-\beta}|f|_{W_{p}^{k+1}},
$$

for every function $f \in W_{p}^{k+1}(\Omega)$ with $0 \leq \alpha+\beta \leq k$. If $\Omega$ is convex then the constant $K_{1}$ depends only on $p, k$ and on the smallest angle $\theta_{\Delta}$ in $\Delta$. If $\Omega$ is non-convex, it also depends on the Lipschitz constant $L_{\partial \Omega}$ associated with the boundary of $\Omega$.

\subsection{A normalized B-spline representation}

Dierckx et al. [6] considered a suitable representation for Powell-Sabin splines. With each vertex $V_{i}$ three linearly independent triplets $\left(\alpha_{i, j}, \beta_{i, j}, \gamma_{i, j}\right), j=1,2,3$ are associated. The B-spline $B_{i}^{j}(x, y)$ can be found as the unique solution of interpolation problem $(2.2)$ with all $\left(f_{l}, f_{x, l}, f_{y, l}\right)=(0,0,0)$ except for $l=i$, where $\left(f_{i}, f_{x, i}, f_{y, i}\right)=\left(\alpha_{i, j}, \beta_{i, j}, \gamma_{i, j}\right) \neq(0,0,0)$. Every Powell-Sabin spline can then be represented as

$$
s(x, y)=\sum_{i=1}^{n} \sum_{j=1}^{3} c_{i, j} B_{i}^{j}(x, y) .
$$

The Powell-Sabin B-splines have a local support: $B_{i}^{j}(x, y)$ vanishes outside the molecule $M_{i}$ of vertex $V_{i}$. The molecule of a vertex (also called vertex star or 1-ring) is the union of all triangles that contain the vertex. The molecule number $m_{i}$ is the number of triangles in $M_{i}$. Dierckx presented in [5] a geometric way to derive and construct triplets $\left(\alpha_{i, j}, \beta_{i, j}, \gamma_{i, j}\right)$ such that the resulting basis forms a convex partition of unity on $\Omega$, i.e.,

$$
B_{i}^{j}(x, y) \geq 0, \quad \text { and } \quad \sum_{i=1}^{n} \sum_{j=1}^{3} B_{i}^{j}(x, y)=1,
$$

for all $(x, y) \in \Omega$. The triplets are found as follows: 
1. For each vertex $V_{i} \in \Delta$, identify the corresponding PS points. These points are defined as the midpoints of all edges in the PS refinement $\Delta^{*}$ containing $V_{i}$. The vertex $V_{i}$ itself is also a PS point. In Figure 1(b) the PS points are indicated as bullets.

2. For each vertex $V_{i}$, find a triangle $t_{i}\left(Q_{i, 1}, Q_{i, 2}, Q_{i, 3}\right)$ containing all the PS points of $V_{i}$. The triangles $t_{i}, i=1, \ldots, n$ are called PS triangles. Note that the PS triangles are not uniquely defined. Figure 1(b) shows some PS triangles. One possibility for their construction [5] is to calculate a triangle of minimal area. The fact that the PS triangle $t_{i}$ contains the PS points of the vertex $V_{i}$ guarantees the positivity property of (2.5).

3. The three linearly independent triplets $\left(\alpha_{i, j}, \beta_{i, j}, \gamma_{i, j}\right), j=1,2,3$ are derived from the PS triangle $t_{i}$ of a vertex $V_{i}$ as follows:

- $\alpha_{i}=\left(\alpha_{i, 1}, \alpha_{i, 2}, \alpha_{i, 3}\right)$ are the barycentric coordinates of $V_{i}$ with respect to $t_{i}$,

- $\beta_{i}=\left(\beta_{i, 1}, \beta_{i, 2}, \beta_{i, 3}\right)$ and $\gamma_{i}=\left(\gamma_{i, 1}, \gamma_{i, 2}, \gamma_{i, 3}\right)$ are the coordinates of the unit barycentric directions, in $x$ - and $y$-direction respectively, with respect to $t_{i}$.

We define the PS control points as $\mathbf{c}_{i, j}=\left(Q_{i, j}, c_{i, j}\right)$. These points define PS control triangles $T_{i}\left(\mathbf{c}_{i, 1}, \mathbf{c}_{i, 2}, \mathbf{c}_{i, 3}\right)$, which are tangent to the spline surface $z=s(x, y)$ at the vertices $V_{i}$. The projection of the control triangles $T_{i}$ in the $(x, y)$-plane are the PS triangles $t_{i}$.

\subsection{Stability of the basis}

From $[16,23]$ we know that the PS B-spline functions form a $L_{\infty}$-stable basis, i.e., for any coefficient vector $C$ in the spline representation (2.4) it holds

$$
K_{2}\|C\|_{\infty} \leq\|s(x, y)\|_{L_{\infty}} \leq\|C\|_{\infty}
$$

where $K_{2}$ only depends on the smallest angle $\theta_{\Delta}$ in the triangulation $\Delta$ and on the size of the PS triangles. Moreover, the smaller the PS triangles the better (the larger) the approximation constant. In [26] it is proved that the B-splines are also stable with respect to a particular weighted $L_{p}$-norm for $p \geq 1$. We give a new proof here which leads to sharper approximation constants. Instead of using generic constants we will denote the considered constants with a successive number, in order to elucidate their propagation in the further theorems.

Theorem 2.2 ( $L_{p}$-stability). The B-spline basis for PS splines in (2.4) is stable with respect to a weighted $L_{p}$-norm where $1 \leq p \leq \infty$, i.e.,

$$
K_{3}\|C\|_{p, w} \leq\|s(x, y)\|_{L_{p}} \leq K_{4}\|C\|_{p, w},
$$

with

$$
\|C\|_{p, w}=\left(\sum_{i=1}^{n} \sum_{j=1}^{3}\left|c_{i, j}\right|^{p} A_{M_{i}}\right)^{1 / p},
$$

and $A_{M_{i}}$ the area of the molecule $M_{i}$ of vertex $V_{i}$. The constants $K_{3}$ and $K_{4}$ only depend on the smallest angle $\theta_{\Delta}$ and on the size of the PS triangles.

Proof. From [26] we know that there exists a constant $K_{5}$ such that

$$
\|s(x, y)\|_{L_{\infty}, \rho} \leq \frac{K_{5}}{A_{\rho}^{1 / p}}\|s(x, y)\|_{L_{p}, \rho},
$$


with $\|\cdot\|_{L_{p}, \rho}$ the $L_{p}$-norm on triangle $\rho \in \Delta$, and $A_{\rho}$ the area of $\rho$. Using (2.6) and (2.9), we get

$$
\|s(x, y)\|_{L_{p}}^{p}=\sum_{\rho \in \Delta}\|s(x, y)\|_{L_{p}, \rho}^{p} \geq \sum_{\rho \in \Delta} \frac{A_{\rho}}{K_{5}{ }^{p}}\|s(x, y)\|_{L_{\infty}, \rho}^{p} \geq \sum_{\rho \in \Delta} \frac{A_{\rho} K_{2}{ }^{p}}{K_{5}{ }^{p}}\|C\|_{\infty, \rho}^{p} .
$$

Since there are nine B-splines non-zero on each triangle $\rho \in \Delta$, and since, by definition, $A_{M_{i}}=$ $\sum_{\left.\rho\right|_{V_{i} \in \rho}} A_{\rho}$, it follows that

$$
\|s(x, y)\|_{L_{p}}^{p} \geq \sum_{i} \sum_{j} \frac{1}{9} \sum_{\left.\rho\right|_{V_{i} \in \rho}} \frac{A_{\rho} K_{2}{ }^{p}}{K_{5}{ }^{p}}\left|c_{i, j}\right|^{p}=\sum_{i} \sum_{j} \frac{A_{M_{i}} K_{2}{ }^{p}}{9 K_{5}{ }^{p}}\left|c_{i, j}\right|^{p},
$$

which proves the lower bound in (2.7) with $K_{3}=9^{-1 / p} K_{2} / K_{5}$.

We now derive the upper bound. Since $0 \leq B_{i}^{j}(x, y) \leq 1$, it holds

$$
\|s(x, y)\|_{L_{p}}^{p}=\sum_{\rho \in \Delta} \int_{\rho}\left|\sum_{\left.i\right|_{V_{i}} \in \rho} \sum_{j} c_{i, j} B_{i}^{j}(x, y)\right|^{p} d \rho \leq \sum_{\rho \in \Delta} \int_{\rho}\left(\sum_{\left.i\right|_{V_{i}} \in \rho} \sum_{j}\left|c_{i, j}\right|\right)^{p} d \rho .
$$

Let $1 / p+1 / q=1$, then by Hölder's inequality for sums,

$$
\begin{aligned}
\|s(x, y)\|_{L_{p}}^{p} & \leq \sum_{\rho \in \Delta}\left(\sum_{\left.i\right|_{V_{i} \in \rho}} \sum_{j}\left|c_{i, j}\right|^{p}\right)\left(\sum_{\left.i\right|_{V_{i} \in \rho}} \sum_{j} 1\right)^{p / q} \int_{\rho} d \rho \\
& =\sum_{\rho \in \Delta} \sum_{\left.i\right|_{V_{i} \in \rho}} \sum_{j}\left|c_{i, j}\right|^{p} 9^{p / q} A_{\rho}=9^{p / q} \sum_{i} \sum_{j} \sum_{\left.\rho\right|_{V_{i} \in \rho}} A_{\rho}\left|c_{i, j}\right|^{p} \\
& =9^{p / q} \sum_{i} \sum_{j} A_{M_{i}}\left|c_{i, j}\right|^{p},
\end{aligned}
$$

which proves the upper bound in (2.7) with $K_{4}=9^{1-1 / p}$.

Let $|\rho|$ be the length of the longest side of triangle $\rho$, and introducing the ratio

$$
K_{6}=\frac{\max _{\rho \in \Delta}|\rho|}{\min _{\rho \in \Delta}|\rho|}
$$

we can also show $L_{p}$-stability for the PS B-spline basis without the weighting used in Theorem 2.2. This result is presented in the following corollary.

Corollary 2.1. The B-spline basis for PS splines is a $L_{p}$-stable basis with $1 \leq p \leq \infty$, i.e., for any coefficient vector $C$ in the PS spline representation (2.4) we have

$$
K_{7}|\Delta|^{2 / p}\|C\|_{p} \leq\|s(x, y)\|_{L_{p}} \leq K_{8}|\Delta|^{2 / p}\|C\|_{p},
$$

with $K_{7}=K_{3}\left(\sin \left(\theta_{\Delta}\right) /\left(4 K_{6}{ }^{2}\right)\right)^{1 / p}$ and $K_{8}=K_{4}\left(\sqrt{3} \pi /\left(2 \theta_{\Delta}\right)\right)^{1 / p}$.

Proof. From elementary geometry we know that the following inequalities hold for any triangle $\rho$ with area $A_{\rho}$ and minimal angle $\theta_{\rho}$ :

$$
\sin \left(\theta_{\rho}\right) \frac{|\rho|^{2}}{4} \leq A_{\rho} \leq \sin \left(\theta_{\rho}\right) \frac{|\rho|^{2}}{2} .
$$




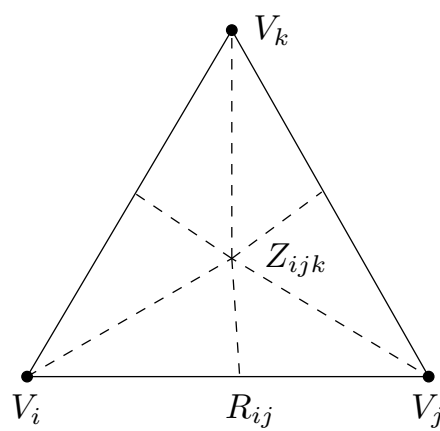

(a)

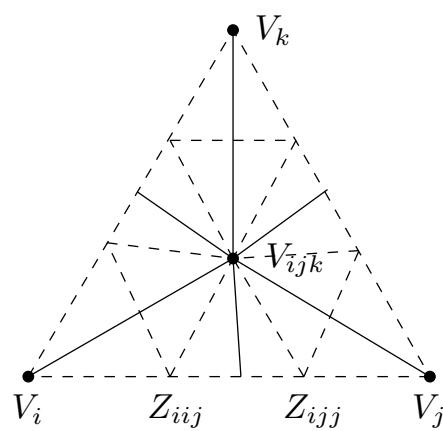

(b)

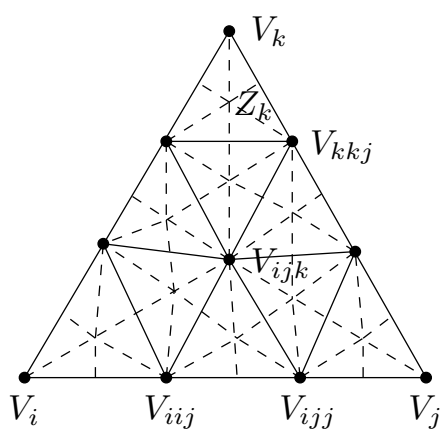

(c)

Figure 2: Two successive $\sqrt{3}$-refinement steps result in a triadic split. The corresponding PS refinement is indicated with dashed lines.

Using (2.12) and the upper bound for the molecule number $m_{i} \leq 2 \pi / \theta_{\Delta}$, we obtain that for any vertex $V_{i}$ in $\Delta$ :

$$
\begin{aligned}
& A_{M_{i}} \leq m_{i} \max _{\rho \in M_{i}} A_{\rho} \leq \frac{\pi}{\theta_{\Delta}} \frac{\sqrt{3}}{2}|\Delta|^{2}, \\
& A_{M_{i}} \geq \max _{\rho \in M_{i}} A_{\rho} \geq \sin \left(\theta_{\Delta}\right) \frac{|\Delta|^{2}}{4 K_{6}{ }^{2}} .
\end{aligned}
$$

Filling (2.13) into (2.8) results in (2.11).

Remark 2.1. Several other stable bases exist on Powell-Sabin triangulations. Their construction is based on so-called minimal determining sets, see e.g. [14, 20].

\subsection{Powell-Sabin spline subdivision}

Subdivision is a procedure to represent a surface on a finer mesh than the mesh on which it is originally defined. The subdivision scheme for Powell-Sabin splines developed in $[27,21]$ is based on the so-called $\sqrt{3}$-refinement scheme of Kobbelt [12], illustrated in Figure 2. The refined triangulation, which we shall denote as $\Delta^{\sqrt{3}}$, is constructed by inserting a new vertex $V_{i j k}$ at the position of the interior point $Z_{i j k}$ inside each triangle $\rho\left(V_{i}, V_{j}, V_{k}\right)$. The edges of the original PS refinement $\Delta^{*}$ that are not edges in the original triangulation $\Delta$ form the edges of the new triangulation $\Delta^{\sqrt{3}}$. The interior points of the new PS refinement are chosen on the edges of $\Delta$. Then, the edges of $\Delta$ will be a subset of the edges of the new PS refinement. Applying the $\sqrt{3}-$ refinement scheme twice, we obtain a triadic split, as shown in Figure 2. Every original edge is trisected and each original triangle is split into nine subtriangles.

The corresponding Powell-Sabin $\sqrt{3}$-subdivision rules are described in [27]. These rules learn how to derive the PS control triangles on the refined mesh, from the given PS control triangles on the original mesh. For the original vertices $V_{i}$ one can reuse the old PS triangles defined by their corner points $Q_{i, m}, m=1,2,3$. However, it is also possible to determine a smaller PS triangle by rescaling the original one through an appropriate scalar $\omega_{i}$. The new corners are given by

$$
Q_{i, m}^{\sqrt{3}}=\omega_{i} V_{i}+\left(1-\omega_{i}\right) Q_{i, m}, \quad m=1,2,3,
$$




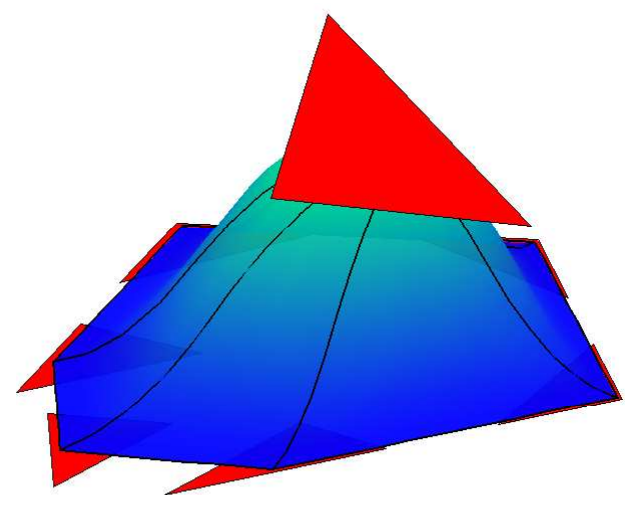

(a)

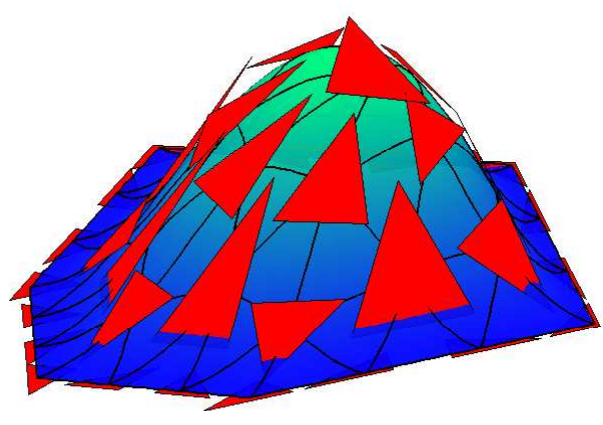

(b)

Figure 3: (a) A PS spline with 7 control triangles; (b) the triadically subdivided spline. The grid lines are drawn on the spline with solid lines.

and the corresponding coefficients $c_{i, m}^{\sqrt{3}}$ are calculated via the old coefficients $c_{i, m}$ as

$$
\begin{aligned}
& c_{i, 1}^{\sqrt{3}}=\left(\omega_{i} \alpha_{i, 1}+1-\omega_{i}\right) c_{i, 1}+\omega_{i} \alpha_{i, 2} c_{i, 2}+\omega_{i} \alpha_{i, 3} c_{i, 3}, \\
& c_{i, 2}^{\sqrt{3}}=\omega_{i} \alpha_{i, 1} c_{i, 1}+\left(\omega_{i} \alpha_{i, 2}+1-\omega_{i}\right) c_{i, 2}+\omega_{i} \alpha_{i, 3} c_{i, 3}, \\
& c_{i, 3}^{\sqrt{3}}=\omega_{i} \alpha_{i, 1} c_{i, 1}+\omega_{i} \alpha_{i, 2} c_{i, 2}+\left(\omega_{i} \alpha_{i, 3}+1-\omega_{i}\right) c_{i, 3} .
\end{aligned}
$$

The PS triangles $t_{i j k}\left(Q_{i j k, 1}^{\sqrt{3}}, Q_{i j k, 2}^{\sqrt{3}}, Q_{i j k, 3}^{\sqrt{3}}\right)$ associated with the new vertices $V_{i j k}$ in the refined mesh are defined by

$$
Q_{i j k, 1}^{\sqrt{3}}=\left(V_{i j k}+V_{i}\right) / 2, \quad Q_{i j k, 2}^{\sqrt{3}}=\left(V_{i j k}+V_{j}\right) / 2, \quad \text { and }, \quad Q_{i j k, 3}^{\sqrt{3}}=\left(V_{i j k}+V_{k}\right) / 2 .
$$

The corresponding coefficients are computed as convex combinations

$$
\begin{aligned}
& c_{i j k, 1}^{\sqrt{3}}=\tilde{L}_{i, 1} c_{i, 1}+\tilde{L}_{i, 2} c_{i, 2}+\tilde{L}_{i, 3} c_{i, 3}, \\
& c_{i j k, 2}^{\sqrt{3}}=\tilde{L}_{j, 1} c_{j, 1}+\tilde{L}_{j, 2} c_{j, 2}+\tilde{L}_{j, 3} c_{j, 3}, \\
& c_{i j k, 3}^{\sqrt{3}}=\tilde{L}_{k, 1} c_{k, 1}+\tilde{L}_{k, 2} c_{k, 2}+\tilde{L}_{k, 3} c_{k, 3} .
\end{aligned}
$$

The triplets $\left(\tilde{L}_{i, 1}, \tilde{L}_{i, 2}, \tilde{L}_{i, 3}\right),\left(\tilde{L}_{j, 1}, \tilde{L}_{j, 2}, \tilde{L}_{j, 3}\right)$ and $\left(\tilde{L}_{k, 1}, \tilde{L}_{k, 2}, \tilde{L}_{k, 3}\right)$ are the barycentric coordinates of $Q_{i j k, 1}^{\sqrt{3}}, Q_{i j k, 2}^{\sqrt{3}}$, and $Q_{i j k, 3}^{\sqrt{3}}$ respectively, with respect to the PS triangles of the surrounding vertices $V_{i}, V_{j}$ and $V_{k}$. Since these points lie inside the corresponding PS triangles, all weights are positive. Applying those rules twice, one obtains the control triangles of the new vertices in a triadically refined mesh, e.g., of vertices $V_{i i j}$ and $V_{i j j}$ in Figure 2(c). Figure 3 illustrates the triadic subdivision for a given PS spline.

Using the triadic subdivision scheme, we can generate a sequence of triangulations $\Delta^{l}$ and corresponding PS refinements $\Delta^{*, l}$ with $l \geq 0$, such that the Powell-Sabin spline spaces $S^{l}=S_{2}^{1}\left(\Delta^{*, l}\right)$ are nested. The superscript $l$ refers to the resolution level, i.e., the number of applied triadic refinements. We denote $P^{l}$ as the subdivision matrix which relates the coefficients of the PS spline on the mesh $\Delta^{l-1}$ to the coefficients of the triadic subdivided PS spline on the finer mesh $\Delta^{l}$. 
Lemma 2.1. Let $\tilde{C}$ be the coefficient vector of the original spline on $\Delta^{l-1}$ and $C$ be the one of the subdivided spline on $\Delta^{l}$, i.e., $C=P^{l} \tilde{C}$. The $l_{p}$-norms, with $1 \leq p \leq \infty$, of both coefficient vectors are related as

$$
K_{9}\|\tilde{C}\|_{p} \leq\|C\|_{p} \leq K_{10}\|\tilde{C}\|_{p},
$$

where $K_{9}=4 / 15 \sin \left(\theta_{\Delta^{*}, l}\right)^{2}$ and $K_{10}=9\left(1 / 3+2 \pi / \theta_{\Delta^{l-1}}\right)^{1 / p}$.

Proof. Throughout the proof we will use the subscripts $i$ and $j$ to refer to coefficients $c_{i, j}$ on the finer mesh $\Delta^{l}$, and the subscripts $g$ and $h$ to refer to coefficients $\tilde{c}_{g, h}$ on the coarser mesh $\Delta^{l-1}$.

We first prove the upper bound. Denote $I_{l}^{l-1}(i)$ as the set of indices of the control triangles $\tilde{T}_{g}$ on mesh $\Delta^{l-1}$ that influence the control triangle $T_{i}$ on mesh $\Delta^{l}$ in the triadic PS subdivision scheme. By (2.14)-(2.15) the elements in the subdivision matrix $P^{l}$ are positive and smaller than 1.

Therefore,

$$
\begin{aligned}
\|C\|_{p}^{p} & =\sum_{i=1}^{n} \sum_{j=1}^{3}\left|c_{i, j}\right|^{p}=\sum_{i=1}^{n} \sum_{j=1}^{3}\left|\sum_{g \in I_{l}^{l-1}(i)} \sum_{h=1}^{3} p_{i, j, g, h}^{l} \tilde{c}_{g, h}\right|^{p} \\
& \leq \sum_{i=1}^{n} \sum_{j=1}^{3}\left|\sum_{g \in I_{l}^{l-1}(i)} \sum_{h=1}^{3} \tilde{c}_{g, h}\right|^{p} \leq \sum_{i=1}^{n} \sum_{j=1}^{3}\left(\sum_{g \in I_{l}^{l-1}(i)} \sum_{h=1}^{3}\left|\tilde{c}_{g, h}\right|\right)^{p} .
\end{aligned}
$$

By Hölder's inequality for sums, we obtain that

$$
\|C\|_{p}^{p} \leq \sum_{i=1}^{n} \sum_{j=1}^{3}\left(\sum_{g \in I_{l}^{l-1}(i)} \sum_{h=1}^{3}\left|\tilde{c}_{g, h}\right|^{p}\right)\left(\sum_{g \in I_{l}^{l-1}(i)} \sum_{h=1}^{3} 1\right)^{p / q}
$$

with $1 / p+1 / q=1$. Because there are at most three indices in each set $I_{l}^{l-1}(i)$, we have

$$
\|C\|_{p}^{p} \leq 9^{p-1} \sum_{i=1}^{n} \sum_{j=1}^{3} \sum_{g \in I_{l}^{l-1}(i)} \sum_{h=1}^{3}\left|\tilde{c}_{g, h}\right|^{p} .
$$

The value $\tilde{c}_{g, h}$ influences the values of the coefficients $c_{i, j}$ corresponding to all vertices $V_{i}$ in the interior of the molecule $M_{g}$ in $\Delta^{l-1}$, with molecule number $m_{g}$. The number of these coefficients $c_{i, j}$ is denoted by $n_{l-1}^{l}(g)$, and it is equal to

$$
n_{l-1}^{l}(g)= \begin{cases}3\left(1+m_{g}+2\left(m_{g}+1\right)\right), & \text { if } V_{g} \in \partial \Delta^{l-1} \\ 3\left(1+m_{g}+2 m_{g}\right), & \text { otherwise }\end{cases}
$$

This number can be bounded as follows: $n_{l-1}^{l}(g) \leq 3\left(1+6 \pi / \theta_{\Delta^{l-1}}\right)$. With this, we can prove the upper bound

$$
\|C\|_{p}^{p} \leq 9^{p-1} 3\left(1+6 \pi / \theta_{\Delta^{l-1}}\right) \sum_{g=1}^{\tilde{n}} \sum_{h=1}^{3}\left|\tilde{c}_{g, h}\right|^{p} .
$$

We now show the lower bound of (2.16). Consider relation (2.14) with, in the notation of this proof, the elements $\tilde{c}_{g, h}$ in the right hand side and $c_{g, j}$ in the left hand side. By inverting the relation, it follows after some algebra that

$$
\left|\tilde{c}_{g, h}\right| \leq \frac{1}{1-\omega_{g}} \sum_{j=1}^{3}\left|c_{g, j}\right|
$$


Note that $\omega_{g}$ stands for the scaling factor after applying twice the $\sqrt{3}$-rule described before, since we consider here the triadic subdivision scheme. With the correct scaling factor the relations (2.14) are also valid for the triadic scheme. For $p \geq 1$ we obtain by Hölder's inequality that

$$
\left|\tilde{c}_{g, h}\right|^{p} \leq \frac{1}{3}\left(\frac{3}{1-\omega_{g}}\right)^{p} \sum_{j=1}^{3}\left|c_{g, j}\right|^{p}
$$

Let $l_{1}$ and $l_{2}$ be two side lengths of the same triangle $\rho$. It holds by the law of sines that

$$
\sin \left(\theta_{\rho}\right) l_{1} \leq l_{2},
$$

with $\theta_{\rho}$ the smallest angle in $\rho$. Referring to Figure 2(c), we know from the construction of the scaling factor $\omega_{g}$ (see [27]) that

$$
1-\omega_{g} \geq \frac{\left\|V_{k}-Z_{k}\right\|_{2}}{\left\|V_{k}-V_{i j k}\right\|_{2}}=\left(1+\frac{\left\|Z_{k}-V_{i j k}\right\|_{2}}{\left\|V_{k}-Z_{k}\right\|_{2}}\right)^{-1} .
$$

Using (2.19) consecutively for the triangles $\rho\left(V_{k}, Z_{k}, V_{k k j}\right)$ and $\rho\left(Z_{k}, V_{i j k}, V_{k k j}\right)$, we obtain

$$
1-\omega_{g} \geq\left(1+\sin \left(\theta_{\Delta^{*}, l}\right)^{-2}\right)^{-1} \geq \frac{4}{5} \sin \left(\theta_{\Delta^{*}, l}\right)^{2} .
$$

The second inequality holds because $\theta_{\Delta^{*}, l} \leq \pi / 6$. Combining (2.18) and (2.21) we find

$$
\|C\|_{p}^{p} \geq \sum_{g=1}^{\tilde{n}} \sum_{j=1}^{3}\left|c_{g, j}\right|^{p} \geq \sum_{g=1}^{\tilde{n}} \sum_{h=1}^{3}\left(\frac{1-\omega_{g}}{3}\right)^{p}\left|\tilde{c}_{g, h}\right|^{p} \geq\left(\frac{4}{15} \sin \left(\theta_{\Delta^{*}, l}\right)^{2}\right)^{p}\|\tilde{C}\|_{p}^{p} .
$$

The stability results of Section 2.3 and the bounds in Lemma 2.1 are derived in terms of the smallest angle in the underlying triangulation $\Delta^{*, l}$. Further on, we will assume that the nested sequence of triangulations is quasi-uniform, i.e., the minimal angle of the generated meshes remains bounded away from zero and the ratio in (2.10) is bounded as well.

\section{Multigrid with Powell-Sabin splines}

\subsection{Multigrid algorithm}

In [22] it is described how Powell-Sabin splines can be used as finite elements to solve the diffusion equation numerically. The variational weak form of (1.1) is given by

$$
a(u, v)=\langle f, v\rangle_{L_{2}}, \quad \forall v \in V=\left\{v \in W_{2}^{1}(\Omega): v=0 \text { on } \partial \Omega\right\},
$$

with $\langle\cdot, \cdot\rangle_{L_{2}}$ the $L_{2}$ scalar product and $a(\cdot, \cdot)$ the energy scalar product on $\Omega$. Denote $u_{*}^{l} \in S^{l}$, with coefficient vector $U_{*}^{l}$, as the Ritz-Galerkin approximation on mesh $\Delta^{l}$ to the solution $u$ of (3.1). Combining Céa's inequality and the Aubin-Nitsche Duality principle (see, e.g. [3]) with the Approximation Theorem 2.1, we can conclude that

$$
\left\|u-u_{*}^{l}\right\|_{L_{2}} \leq K_{11}\left|\Delta^{l}\right|^{k+1}|u|_{W_{2}^{k+1}}, \quad \text { for } \quad 0 \leq k \leq 2,
$$

and using elliptic regularity we get

$$
\left\|u-u_{*}^{l}\right\|_{L_{2}} \leq K_{12}\left|\Delta^{l}\right|^{2}\|f\|_{L_{2}}
$$


with $K_{11}$ and $K_{12}$ independent of the mesh size of $\Delta^{l}$. Further on, to simplify our notation, we will omit the superscript $l$ for the variables corresponding to the finest level. We will denote the splines and coefficient vectors corresponding to the coarser mesh $\Delta^{l-1}$ with a tilde.

The discretization of (3.1) with Powell-Sabin spline finite elements leads to the sparse linear system $[22]$

$$
G U_{*}=F,
$$

where $G$ is called the stiffness matrix. Simple iterative schemes, like the Gauss-Seidel method, converge very slowly for large such systems. The finer the mesh size, the slower the convergence of these methods. The idea of a multigrid method is to use calculations on coarser grids to accelerate the convergence on the fine grid. Basically, a multigrid method consists of two steps. With a classical simple iteration scheme the high frequency components of the error are quickly removed (smoothing step). The remaining error can be well approximated by solving recursively similar problems on coarser grids (coarse grid correction step). Algorithm 3.1 gives a schematic overview of a multigrid iteration. Note that the stiffness matrix $\tilde{G}$ defined on the coarser mesh $\Delta^{l-1}$ is related to the one on the finer mesh $\Delta^{l}$ as $\tilde{G}=P^{T} G P$, with $P$ the PS subdivision matrix.

Algorithm 3.1 (Multigrid). An iteration of the $\gamma$-cycle multigrid scheme for improving the initial approximation $U_{0}$ to the solution $U_{*}=G^{-1} F$ on level $l>0$, is defined as

$$
\begin{aligned}
& \text { function } U_{M G}=M G\left(U_{0}, F, l\right) \\
& U_{S^{\nu}}=S^{\nu_{\text {pre }}}\left(U_{0}, F\right) \\
& R=G U_{S^{\nu}}-F, \quad \tilde{R}=P^{T} R \\
& \text { if } l>1 \text { then } \\
& \tilde{U}_{M G}=M G^{\gamma}(0, \tilde{R}, l-1) \\
& \text { else } \\
& \text { solve } \tilde{G} \tilde{U}_{M G}=\tilde{R} \\
& \text { end } \\
& U_{M G}=S^{\nu_{\text {post }}}\left(U_{S^{\nu}}-P \tilde{U}_{M G}, F\right)
\end{aligned}
$$

with $\left(\nu_{\text {pre }}, \nu_{\text {post }}\right)$ the number of smoothing iterations, and $\gamma$ the number of coarse grid iterations. The cases $\gamma=1$ and $\gamma=2$ are known as the multigrid $V$-cycle and $W$-cycle respectively.

To simplify the convergence analysis of the multigrid method we will use a damped Richardson iteration scheme as smoother, as will be discussed in section 3.2. A good initial approximation $U_{0}$ can be found by subdividing (a multigrid approximation of) the solution of the system $\tilde{G} \tilde{U}_{*}=\tilde{F}$ on a coarser grid. Repeating that for all levels, one obtains the full multigrid algorithm.

\subsection{Smoothing step}

As smoothing scheme we choose the Richardson method, which updates an approximation $U_{0}$ to the solution $U_{*}=G^{-1} F$ of the Galerkin system (3.4) by adding a multiple of the residual,

$$
U_{S}=S\left(U_{0}, F\right)=U_{0}-\lambda^{-1}\left(G U_{0}-F\right) .
$$

For instance, we may choose the damping factor $\lambda$ as

$$
\lambda=\|G\|_{\infty}=\max _{i} \sum_{j}\left|g_{i, j}\right|,
$$

i.e., an upper bound for the largest eigenvalue of $G$. The next lemma shows that this choice of $\lambda$ can be bounded above by a constant independent of the mesh size $\left|\Delta^{l}\right|$. 
Lemma 3.1. The max-norm of the stiffness matrix $G$ in (3.4) is bounded by a constant independent of the mesh size $\left|\Delta^{l}\right|$, i.e., $\|G\|_{\infty} \leq K_{14}$.

Proof. The inradius $r_{\rho}$ of a triangle $\rho$ can be written as

$$
r_{\rho}=\tan \left(\frac{\theta_{3}}{2}\right) \frac{l_{1}+l_{2}-l_{3}}{2},
$$

with $l_{i}$ the lengths of the sides of $\rho$, and $l_{3}$ corresponds to the side opposite to angle $\theta_{3}$. Let $|\rho|$ be the longest side length and $\theta_{\rho}$ the smallest angle in $\rho$, then the inradius can be bounded below by

$$
r_{\rho} \geq \tan \left(\frac{\theta_{\rho}}{2}\right) \frac{|\rho|}{2} .
$$

Combining (2.12) with (3.7), we obtain that

$$
A_{\rho} \leq \frac{2 \sin \left(\theta_{\rho}\right)}{\tan \left(\theta_{\rho} / 2\right)^{2}} r_{\rho}^{2}=\frac{4 \cos \left(\theta_{\rho} / 2\right)^{2}}{\tan \left(\theta_{\rho} / 2\right)} r_{\rho}^{2} \leq \frac{4 r_{\rho}^{2}}{\tan \left(\theta_{\rho} / 2\right)} .
$$

Consider a triangle $\rho^{*} \in \Delta^{*, l}$. From [16] we know that for $s \in S^{l}$

$$
\left\|D_{x} s\right\|_{L_{\infty}, \rho^{*}} \leq \frac{12}{r_{\rho^{*}}}\|s\|_{L_{\infty}, \rho^{*}}, \quad \text { and } \quad\left\|D_{y} s\right\|_{L_{\infty}, \rho^{*}} \leq \frac{12}{r_{\rho^{*}}}\|s\|_{L_{\infty}, \rho^{*}}
$$

Suppose that the PS B-splines $B_{i}^{j}(x, y)$ and $B_{g}^{h}(x, y)$ are both non-zero on $\rho^{*}$. Using (3.8), (3.9) and the partition of unity of the PS basis, it follows that

$$
\left|a\left(B_{i}^{j}(x, y), B_{g}^{h}(x, y)\right)_{\rho *}\right| \leq 2 A_{\rho *}\left(\frac{12}{r_{\rho *}}\right)^{2} \leq 8 \frac{12^{2}}{\tan \left(\theta_{\Delta^{*}, l} / 2\right)}=K_{13},
$$

with $a(\cdot, \cdot)_{\rho *}$ the energy scalar product restricted to $\rho *$. We now count how many triangles $\rho^{*} \in \Delta^{*, l}$ are shared by the support of $B_{i}^{j}(x, y)$ corresponding to vertex $V_{i}$ and the support of the other PS Bsplines. The three PS B-splines associated with $V_{i}$ have $6 m_{i}$ triangles in common. For the other Bsplines we need to distinguish two cases dependent on the position of the vertex $V_{i}$. If $V_{i}$ is situated at the boundary of the triangulation, then there are $3\left(m_{i}-1\right)$ PS B-splines with 12 triangles in common, and 6 PS B-splines with 6 triangles in common. If instead $V_{i}$ is an interior vertex, then there are $3 m_{i}$ PS B-splines that have 12 triangles in common. The remaining B-splines have no triangles in common. Since the molecule number $m_{i}$ can be bounded by $m_{i} \leq 2 \pi / \theta_{\Delta^{l}}$, we obtain that $K_{14}=108 \pi / \theta_{\Delta^{l}} K_{13}$, which is only dependent on the smallest angle in the triangulation.

The error $U_{S^{\nu}}-U_{*}$ after $\nu$ Richardson steps with $U_{S^{\nu}}=S^{\nu}\left(U_{0}, F\right)$ is equal to

$$
U_{S^{\nu}}-U_{*}=\left(I-\lambda^{-1} G\right)^{\nu}\left(U_{0}-U_{*}\right) .
$$

We now give a relation between the $l_{2}$-norms of the residual $R=G U_{S^{\nu}}-F=G\left(U_{S^{\nu}}-U_{*}\right)$ and the initial error $U_{0}-U_{*}$.

Theorem 3.1 (Richardson's smoothing). The residual $R=G\left(U_{S^{\nu}}-U_{*}\right)$ after $\nu$ Richardson steps is related to the initial error $U_{0}-U_{*}$ as

$$
\|R\|_{2} \leq \frac{K_{14}}{e \sqrt{\nu(\nu+1)}}\left\|U_{0}-U_{*}\right\|_{2} .
$$

with $K_{14}$ independent of the mesh size $\left|\Delta^{l}\right|$ and the number of smoothing steps $\nu$. 
Proof. The inequality is proved by estimating the eigenvalues $\hat{\lambda}_{i}$ of the symmetric matrix

$$
G\left(I-\lambda^{-1} G\right)^{\nu},
$$

i.e. the transition matrix from $U_{0}-U_{*}$ to $R$. By (3.6) we know that $\tau_{i}=\lambda_{i} / \lambda \in(0,1]$ for any eigenvalue $\lambda_{i}$ of $G$. Hence,

$$
\left|\hat{\lambda}_{i}\right| \leq \max _{\tau \in[0,1]} \lambda \tau(1-\tau)^{\nu}=\frac{\lambda}{1+\nu}\left(\frac{\nu}{1+\nu}\right)^{\nu} .
$$

Using the following bound for the exponential function [11],

$$
e^{x} \leq\left(1+\frac{x}{t}\right)^{t+x / 2}
$$

with $t=\nu$ and $x=1$, we have

$$
\left|\hat{\lambda}_{i}\right| \leq \frac{\lambda}{e \sqrt{\nu(1+\nu)}} .
$$

By Lemma 3.1 we obtain the desired bound.

\subsection{Convergence of the multigrid scheme}

In this section we will show that the proposed multigrid scheme converges with respect to the $l_{2}$-norm, with a convergence factor that is bounded independent of the mesh size $\left|\Delta^{l}\right|$. The convergence rate is established based on the theory in [9]. The derivation can be slightly simplified in case of nested Powell-Sabin spline spaces. We first consider the analysis of a two-grid scheme.

Theorem 3.2 (Coarse grid correction). Let $R=G\left(U_{S^{\nu}}-U_{*}\right)$ be the residual on the mesh $\Delta^{l}$ of approximation $U_{S^{\nu}}$, and $\tilde{R}=P^{T} R$ be its restriction on the coarser mesh $\Delta^{l-1}$. Let $U_{2 G}=U_{S^{\nu}}-$ $P \tilde{U}_{*}$ be the approximation of the coarse grid correction, with $\tilde{U}_{*}$ the exact solution of $\tilde{G} \tilde{U}_{*}=\tilde{R}$, then

$$
\left\|U_{2 G}-U_{*}\right\|_{2} \leq K_{15}\|R\|_{2},
$$

where $K_{15}$ is independent of the mesh size $\left|\Delta^{l}\right|$.

Proof. Denote $u_{S^{\nu}}$ and $u_{*}$ as the PS splines on $\Delta^{l}$ corresponding to the coefficient vectors $U_{S^{\nu}}$ and $U_{*}$ respectively. Let $\tilde{u}_{*}$ be the PS spline on $\Delta^{l-1}$ corresponding to the coefficient vector $\tilde{U}_{*}$. By the definitions of the coefficient vectors $R=G\left(U_{S^{\nu}}-U_{*}\right)$ and $\tilde{R}=\tilde{G} \tilde{U}_{*}$, we know that their elements $r_{i, j}$ and $\tilde{r}_{g, h}$ are given by

$$
r_{i, j}=a\left(u_{S^{\nu}}-u_{*}, B_{i}^{j}\right), \quad \text { and } \quad \tilde{r}_{g, h}=a\left(\tilde{u}_{*}, \tilde{B}_{g}^{h}\right) .
$$

Let $I_{l-1}^{l}(g)$ be the set of indices of the control triangles $T_{i}$ on mesh $\Delta^{l}$ that are influenced by the control triangle $\tilde{T}_{g}$ on mesh $\Delta^{l-1}$ in the triadic PS subdivision scheme. Since $\tilde{R}=P^{T} R$, it follows

$$
\begin{aligned}
\tilde{r}_{g, h} & =\sum_{i \in I_{l-1}^{l}(g)} \sum_{j=1}^{3} p_{i, j, g, h}^{l} r_{i, j}=\sum_{i \in I_{l-1}^{l}(g)} \sum_{j=1}^{3} p_{i, j, g, h}^{l} a\left(u_{S^{\nu}}-u_{*}, B_{i}^{j}\right) \\
& =a\left(u_{S^{\nu}}-u_{*}, \sum_{i \in I_{l-1}^{l}(g)} \sum_{j=1}^{3} p_{i, j, g, h}^{l} B_{i}^{j}\right)=a\left(u_{S^{\nu}}-u_{*}, \tilde{B}_{g}^{h}\right) .
\end{aligned}
$$

Combining (3.18) and (3.19), we get

$$
a\left(\tilde{u}_{*}, \tilde{B}_{g}^{h}\right)=a\left(u_{S^{\nu}}-u_{*}, \tilde{B}_{g}^{h}\right) .
$$


We now define the function $v$ as the solution of an auxiliary Poisson problem

$$
-\Delta v=q \text { in } \Omega,
$$

with $v=0$ on $\partial \Omega$, and $q \in S^{l}$ the unique PS spline determined by

$$
\langle q, s\rangle_{L_{2}}=a\left(u_{S^{\nu}}-u_{*}, s\right), \quad \forall s \in S^{l} .
$$

By (3.20) and (3.22) it holds for all basis functions $B_{i}^{j} \in S^{l}$ and $\tilde{B}_{g}^{h} \in S^{l-1} \subset S^{l}$ that

$$
\left\langle q, B_{i}^{j}\right\rangle_{L_{2}}=a\left(u_{S^{\nu}}-u_{*}, B_{i}^{j}\right), \quad \text { and }\left\langle q, \tilde{B}_{g}^{h}\right\rangle_{L_{2}}=a\left(\tilde{u}_{*}, \tilde{B}_{g}^{h}\right) .
$$

Therefore, $u_{S^{\nu}}-u_{*}$ and $\tilde{u}_{*}$ are both Ritz-Galerkin approximations to $v$. Using the standard error estimate (3.3) and the following inequality on the mesh sizes,

$$
\left|\Delta^{l-1}\right| \leq 3\left|\Delta^{l}\right|
$$

it follows that

$$
\left\|u_{S^{\nu}}-u_{*}-\tilde{u}_{*}\right\|_{L_{2}} \leq\left\|u_{S^{\nu}}-u_{*}-v\right\|_{L_{2}}+\left\|v-\tilde{u}_{*}\right\|_{L_{2}} \leq 10 K_{12}\left|\Delta^{l}\right|^{2}\|q\|_{L_{2}} .
$$

By the definition of $q$ in (3.22), with coefficient vector $Q$, we get

$$
\|q\|_{L_{2}}^{2}=a\left(u_{S^{\nu}}-u_{*}, q\right)=Q^{T} G\left(U_{S^{\nu}}-U_{*}\right) \leq\|Q\|_{2}\|R\|_{2},
$$

and by (2.11), we obtain

$$
\begin{aligned}
& \|Q\|_{2} \leq\left(K_{7}\left|\Delta^{l}\right|\right)^{-1}\|q\|_{L_{2}}, \\
& \left\|U_{2 G}-U_{*}\right\|_{2} \leq\left(K_{7}\left|\Delta^{l}\right|\right)^{-1}\left\|u_{S^{\nu}}-u_{*}-\tilde{u}_{*}\right\|_{L_{2}} .
\end{aligned}
$$

Combining (3.25), (3.26) and (3.27), the proof is completed with $K_{15}=10 K_{12} / K_{7}{ }^{2}$.

Theorem 3.3 (Multigrid convergence). Let $U_{M G}$ be the solution after a $\gamma$-cycle multigrid iteration (see Algorithm 3.1) with $\gamma>1$ and no post-smoothing steps. There exists a constant $0<\varrho<1$ independent of the mesh size $\Delta^{l}$, such that

$$
\left\|U_{M G}-U_{*}\right\|_{2} \leq \varrho\left\|U_{0}-U_{*}\right\|_{2},
$$

for a sufficiently large (but mesh size independent) number of Richardson's pre-smoothing steps $\nu$.

Proof. Combining Theorems 3.1 and 3.2, we obtain convergence for the two-grid scheme,

$$
\left\|U_{2 G}-U_{*}\right\|_{2} \leq \frac{K_{14} K_{15}}{e \sqrt{\nu(\nu+1)}}\left\|U_{0}-U_{*}\right\|_{2}=K_{16}\left\|U_{0}-U_{*}\right\|_{2},
$$

where $K_{16}<1$ when $\nu(\nu+1)>\left(K_{14} K_{15} / e\right)^{2}$.

We prove the general case with more than two grids via induction. We split the error of the approximation $U_{M G}=U_{S^{\nu}}-P \tilde{U}_{M G}$ after one multigrid step in the form

$$
\left\|U_{M G}-U_{*}\right\| \leq\left\|U_{S^{\nu}}-P \tilde{U}_{*}-U_{*}\right\|_{2}+\left\|P\left(\tilde{U}_{*}-\tilde{U}_{M G}\right)\right\|_{2} .
$$

The first term is bounded by (3.29), and the second term is bounded by Lemma 2.1 and the induction hypothesis, i.e.,

$$
\left\|U_{M G}-U_{*}\right\| \leq K_{16}\left\|U_{0}-U_{*}\right\|_{2}+K_{10} \varrho^{\gamma}\left\|\tilde{U}_{*}\right\|_{2} .
$$


It holds that

$$
K_{9}\left\|\tilde{U}_{*}\right\|_{2} \leq\left\|P \tilde{U}_{*}\right\|_{2} \leq\left\|P \tilde{U}_{*}-U_{S^{\nu}}+U_{*}\right\|_{2}+\left\|U_{S^{\nu}}-U_{*}\right\|_{2},
$$

and since Richardson's method reduces the norm of the error

$$
\left\|\tilde{U}_{*}\right\|_{2} \leq \frac{K_{16}+1}{K_{9}}\left\|U_{0}-U_{*}\right\|_{2} .
$$

Combining (3.30) and (3.31), we obtain

$$
\left\|U_{M G}-U_{*}\right\| \leq\left(K_{16}+\varrho^{\gamma} \frac{K_{10}}{K_{9}}\left(K_{16}+1\right)\right)\left\|U_{0}-U_{*}\right\|_{2} .
$$

For any $\varrho<\left(K_{9} / K_{10}\right)^{1 /(\gamma-1)}$, one can choose the number of smoothing steps $\nu$ such that

$$
K_{16} \leq \frac{\varrho\left(1-\varrho^{\gamma-1} K_{10} / K_{9}\right)}{1+\varrho^{\gamma} K_{10} / K_{9}} \leq \varrho
$$

or

$$
\nu(\nu+1) \geq\left(\frac{K_{14} K_{15}}{e \varrho}\right)^{2},
$$

then inequality (3.28) holds.

We now show that the multigrid spline approximation, obtained by applying the full multigrid scheme, will converge to the exact solution of (3.1) with the same rate as the Ritz-Galerkin spline approximation, i.e. (3.2). The proof is adopted from [3].

Theorem 3.4 (Full multigrid). Let $u_{F M G}$ be the PS spline obtained after a full multigrid step with $\delta$ multigrid iterations on each level. If $\delta$ is large enough then

$$
\left\|u_{F M G}-u_{*}\right\|_{L_{2}} \leq K_{17}\left|\Delta^{l}\right|^{k+1}|u|_{W_{2}^{k+1}}, \quad \text { for } \quad 0 \leq k \leq 2,
$$

with $K_{17}$ independent of level $l$ and mesh size $\left|\Delta^{l}\right|$.

Proof. By (2.11) and (3.28) there exists a constant $0<\hat{\varrho}<1$, such that

$$
\left\|u_{M G}-u_{*}\right\|_{L_{2}} \leq \hat{\varrho}\left\|u_{0}-u_{*}\right\|_{L_{2}},
$$

with $u_{0}$ the initial spline approximation to the spline $u_{*}$. Using (3.2), (3.24) and (3.35) we obtain

$$
\begin{aligned}
\left\|u_{F M G}-u_{*}\right\|_{L_{2}} & \leq \hat{\varrho}^{\delta}\left\|\tilde{u}_{F M G}-u_{*}\right\|_{L_{2}} \\
& \leq \hat{\varrho}^{\delta}\left(\left\|\tilde{u}_{F M G}-\tilde{u}_{*}\right\|_{L_{2}}+\left\|\tilde{u}_{*}-u\right\|_{L_{2}}+\left\|u-u_{*}\right\|_{L_{2}}\right) \\
& \leq \hat{\varrho}^{\delta}\left(\left\|\tilde{u}_{F M G}-\tilde{u}_{*}\right\|_{L_{2}}+\left(1+3^{k+1}\right) K_{11}\left|\Delta^{l}\right|^{k+1}|u|_{W_{2}^{k+1}}\right) .
\end{aligned}
$$

By iterating the above inequality on all levels, it follows that

$$
\begin{aligned}
\left\|u_{F M G}-u_{*}\right\|_{L_{2}} & \leq\left(1+3^{k+1}\right) K_{11}\left|\Delta^{l}\right|^{k+1}|u|_{W_{2}^{k+1}}\left(\varrho^{\delta}+\hat{\varrho}^{2 \delta} 3^{k+1}+\ldots+\hat{\varrho}^{l \delta} 3^{(k+1)(l-1)}\right) \\
& \leq \frac{\hat{\varrho}^{\delta}\left(1+3^{k+1}\right) K_{11}}{1-\hat{\varrho}^{\delta} 3^{k+1}}\left|\Delta^{l}\right|^{k+1}|u|_{W_{2}^{k+1}},
\end{aligned}
$$

for $\varrho^{\delta}<3^{-k-1}$.

Remark 3.1. The number of non-zero elements in the stiffness, smoothing and subdivision matrices of level $l$ are proportional to the number of unknowns $N^{l}$. Since $N^{l-1}<N^{l} / 9$, both the memory and computational cost to obtain $u_{F M G}$ are $\mathcal{O}\left(N^{l}\right)$ for each $\gamma<9$. 


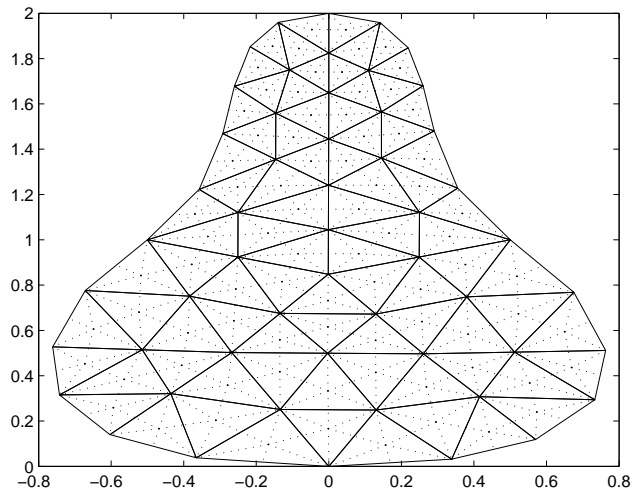

(a) Initial triangulation

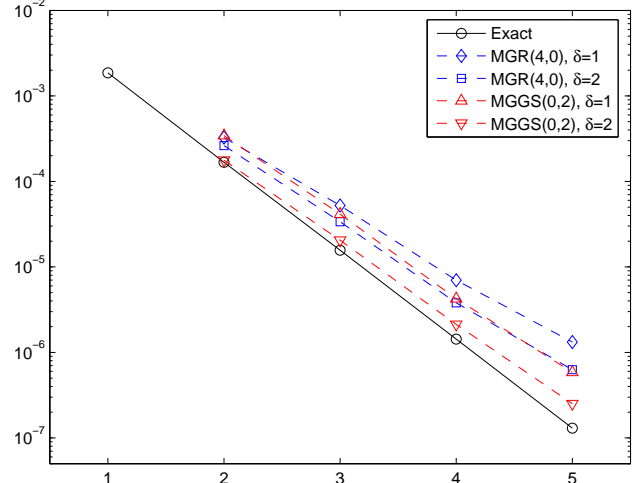

(b) Convergence of error versus number of levels

Figure 4: (a) Initial triangulation with PS refinement. (b) Convergence of the error in the $L_{2^{-}}$ norm for the exactly solved Ritz-Galerkin solution compared with full W-cycle multigrid solutions, using Richarson (MGR) or Gauss-Seidel (MGGS) smoothing. The pair $\left(\nu_{\text {pre }}, \nu_{\text {post }}\right)$ stands for the number of pre- and post-smoothing steps, and $\delta$ is the number of $\mathrm{W}$-cycle multigrid iterations on each level.

\begin{tabular}{|c|r|ccc|ccc|}
\hline & & \multicolumn{3}{|c|}{ Richardson } & \multicolumn{3}{|c|}{ Gauss-Seidel } \\
level & $\operatorname{dim}$ & $(1,0)$ & $(2,0)$ & $(4,0)$ & $(0,1)$ & $(0,2)$ & $(0,4)$ \\
\hline 2 & 975 & 0.88 & 0.79 & 0.69 & 0.54 & 0.33 & 0.20 \\
3 & 9399 & 0.79 & 0.70 & 0.60 & 0.50 & 0.40 & 0.29 \\
4 & 86511 & 0.72 & 0.63 & 0.55 & 0.47 & 0.40 & 0.30 \\
5 & 784407 & 0.69 & 0.60 & 0.54 & 0.44 & 0.38 & 0.31 \\
\hline
\end{tabular}

Table 1: The convergence factor $\varrho(1,4)$, as defined in (3.36), for W-cycle multigrid schemes with Richardson and Gauss-Seidel smoothing. The pair $\left(\nu_{\text {pre }}, \nu_{\text {post }}\right)$ stands for the number of pre- and post-smoothing steps. The first column denotes the number of levels, and the second column shows the dimension of the stiffness matrix.

\subsection{Numerical experiments}

We numerically solve the Poisson equation (1.1) with right-hand side $f=\sin (\pi x) \sin (\pi y)$ on a pear-shaped domain. The initial triangulation is shown in Figure 4(a), and the finer triangulations are obtained using a triadic refinement. The discretized system (3.4) is iteratively solved using Algorithm 3.1 with Richardson and Gauss-Seidel smoothing. Note that Jacobi smoothing does not converge for the stiffness matrix constructed with Powell-Sabin spline finite elements.

In Table 1 we show the convergence factors of some $\mathrm{W}$-cycle multigrid schemes. Each convergence factor is approximated as

$$
\varrho(i, j)=\left(\frac{\left\|U_{M G}^{j}-U_{*}\right\|_{2}}{\left\|U_{M G}^{i}-U_{*}\right\|_{2}}\right)^{1 /(j-i)} \quad, \quad \text { with } j>i,
$$

where $U_{M G}^{j}$ is the multigrid solution after $j$ multigrid iterations. The initial solution $U_{0}$ is taken to be the zero vector. There is no essential difference in the convergence factor, if we replace some Richardson pre-smoothing steps by post-smoothing steps. However, with the Gauss-Seidel scheme 
the best convergence factors are obtained when more post-smoothing than pre-smoothing steps are taken. Table 1 confirms that the proposed multigrid schemes converge independently of the mesh size of the triangulations.

In Figure 4(b) we compare the $L_{2}$-norm of the error $\left\|u-u_{*}\right\|_{L_{2}}$ when we solve system (3.4) exactly on successively refined meshes, to the one obtained by applying a full multigrid iteration scheme, i.e. $\left\|u-u_{F M G}\right\|_{L_{2}}$, with 1 and $2 \mathrm{~W}$-cycle multigrid iterations used on each level. We see that the full multigrid spline approximations $u_{F M G}$ converge almost as quickly as the exact Ritz-Galerkin spline approximation $u^{*}$ to the solution $u$.

\section{Concluding remarks}

The Powell-Sabin B-splines have some favorable properties when used as finite element basis functions on triangulations. Thanks to the high continuity of the quadratic splines, and the low dimension of the spline space, the Ritz-Galerkin approximation converges rapidly to the solution of an elliptic partial differential equation for a reasonably low number of degrees of freedom. A multigrid scheme can be used to efficiently solve such a discretized system. Based on the triadic subdivision scheme for PS splines, very natural grid transfer operators can be constructed.

We proved that the multigrid method with Powell-Sabin splines converges uniformly in the $l_{2}$-norm, i.e., the number of required iterations is independent of the mesh size. This was confirmed with a numerical experiment. The analysis in Section 3.3 should serve as a simple multigrid example. Basically, we may expect that most of the results typical for common finite elements will remain valid for Powell-Sabin splines. We described the method for the Poisson equation, but it is clear that the approach applies to more general second order elliptic problems as well.

\section{References}

[1] P. Alfeld and L.L. Schumaker. The dimension of bivariate spline spaces of smoothness $r$ for degree $d \geq 4 r+1$. Constr. Approx., 3:189-197, 1987.

[2] J.H Bramble. Multigrid methods. Pitman Research Notes in Mathematics 294. John Wiley and Sons, 1993.

[3] S.C. Brenner and L.R. Scott. The mathematical theory of finite element methods. SpringerVerlag, New York, second edition, 2002.

[4] R.W. Clough and J.L. Tocher. Finite element stiffness matrices for analysis of plates in bending. In Proc. 1st Conf. on Matrix Methods in Structural Mechanics, pages 515-545, Wright Patterson Air Force Base, Ohio, 1965.

[5] P. Dierckx. On calculating normalized Powell-Sabin B-splines. Comput. Aided Geom. Design, 15(1):61-78, 1997.

[6] P. Dierckx, S. Van Leemput, and T. Vermeire. Algorithms for surface fitting using PowellSabin splines. IMA J. Numer. Anal., 12:271-299, 1992.

[7] W. Hackbusch. Multigrid methods and applications. Springer-Verlag, Berlin, 1985.

[8] Y. He, M. Jin, X. Gu, and H. Qin. A $C^{1}$ globally interpolatory spline of arbitrary topology. In N. Paragios, O.D. Faugeras, T. Chan, and C. Schnörr, editors, Proc. 3rd IEEE Workshop on Variational, Geometric and Level Set Methods in Computer Vision, pages 295-306, Beijing, China, 2005. 
[9] K. Höllig, U. Reif, and J. Wipper. Multigrid methods with web-splines. Numer. Math., 91:237-256, 2002.

[10] D. Hong. Spaces of bivariate spline functions over triangulation. Approx. Theory Appl., 7:56-75, 1991.

[11] S.H. Kim. Densely algebraic bounds for the exponential function. Proc. Amer. Math. Soc., $135: 237-241,2007$.

[12] L. Kobbelt. $\sqrt{3}$-Subdivision. In Computer Graphics Proceedings, Annual Conference Series, pages 103-112. ACM SIGGRAPH, 2000.

[13] M.J. Lai and L.L. Schumaker. On the approximation power of bivariate splines. Adv. Comp. Math., 9:251-279, 1998.

[14] M.J. Lai and L.L. Schumaker. Macro-elements and stable local bases for spaces of splines on Powell-Sabin triangulations. Math. Comp., 72:335-354, 2003.

[15] J. Maes and A. Bultheel. $C^{1}$ hierarchical Riesz bases of Lagrange type on Powell-Sabin triangulations. J. Comput. Appl. Math., 196(1):1-19, 2006.

[16] J. Maes, E. Vanraes, P. Dierckx, and A. Bultheel. On the Stability of normalized Powell-Sabin B-splines. J. Comput. Appl. Math., 170(1):181-196, 2004.

[17] C. Manni. Shape control in Powell-Sabin quasi-interpolation. In A. Iske and J. Levesley, editors, Algorithms for Approximation, pages 219-240, Heidelberg, 2006. Springer-Verlag.

[18] P. Oswald. Hierarchical conforming finite element methods for the biharmonic equation. SIAM J. Numer. Anal., 29(6):1610-1625, 1992.

[19] M.J.D. Powell and M.A. Sabin. Piecewise quadratic approximations on triangles. ACM Trans. Math. Softw., 3:316-325, 1977.

[20] L.L. Schumaker and T. Sorokina. Smooth macro-elements on Powell-Sabin-12 splits. Math. Comp., 75:711-726, 2006.

[21] H. Speleers, P. Dierckx, and S. Vandewalle. Local subdivision of Powell-Sabin splines. Comput. Aided Geom. Design, 23(5):446-462, 2006.

[22] H. Speleers, P. Dierckx, and S. Vandewalle. Numerical solution of partial differential equations with Powell-Sabin splines. J. Comput. Appl. Math., 189(1-2):643-659, 2006.

[23] H. Speleers, P. Dierckx, and S. Vandewalle. Quasi-hierarchical Powell-Sabin B-splines. Technical Report 472, Dept. Comp. Science, K.U. Leuven, 2006.

[24] G. Strang. Piecewise polynomials and the finite element method. Bull. Amer. Math. Soc., 79:1128-1137, 1973.

[25] E. Vanraes and A. Bultheel. Modelling sharp features with tangent subdivision. In M. Dæhlen, K. Mörken, and L.L. Schumaker, editors, 6th Int. Conf. on Mathematical Methods for Curves and Surfaces, pages 362-372. Nashboro Press, 2005.

[26] E. Vanraes, J. Maes, and A. Bultheel. Powell-Sabin spline wavelets. Int. J. Wav. Multires. Inf. Proc., 2(1):23-42, 2004.

[27] E. Vanraes, J. Windmolders, A. Bultheel, and P. Dierckx. Automatic construction of control triangles for subdivided Powel-Sabin splines. Comput. Aided Geom. Design, 21(7):671-682, 2004.

[28] K. Willemans and P. Dierckx. Smoothing scattered data with a monotone Powell-Sabin spline surface. Numer. Algorithms, 12:215-232, 1996. 